\section{The Royal College of Ophthalmologists' clinical guidelines for diabetic retinopathy: a summary}

\author{
Introduction \\ Incidence of diabetes mellitus (DM) is increasing \\ globally. Around $90 \%$ of the burden is caused by type II \\ diabetes, a preventable chronic disease. Approximately \\ $4.56 \%$ of the UK population is diabetic, totalling just \\ under $1.4 \mathrm{~m}$. The incidence of both type I and type II \\ diabetes is on the rise. Diabetic retinopathy (DR) is a \\ microvascular complication of DM. Both the duration of \\ diabetes and glycaemic control are independent risk \\ factors for severity and progression of DR. DR is more \\ prevalent in older age groups with long-standing \\ disease.
}

\section{Epidemiology}

Many studies exist on diabetic eye disease in different parts of the world, all of which provide a picture of increasing concern with respect to the prevalence of this disorder. The burden of retinopathy is significant, certain epidemiological characteristics are documented.

Younger-onset group of diabetic patients have twice more prevalence of proliferative diabetic retinopathy (PDR) compared with the older-onset group that takes insulin. The 10-year incidence of macular oedema is reportedly two-fold higher in insulin-dependent diabetic population compared with non-insulin-dependent diabetic population. Certain patient groups such as the South Asian or Latinos have a higher prevalence of DR and maculopathy. In children with type I DM, retinopathy rates increase two fold after duration of 6 years. Type II DM is on the rise in young people, usual onset being around age of puberty. This is of concern as the rates of retinopathy may be higher in type II DM than type I in younger patients, and that too with shorter duration. Emerging data suggest that improved standard of care has positive impact on incidence of diabetic retinopathy.

\section{Classification}

The classification of DR has a dichotomous approachthe presence or absence of new vessels (PDR), the presence or absence of centre-involving/subfoveal macular oedema and/or ischaemia. Such features assist in evaluation of the risk of imminent visual impairment (centre-involving macular oedema, new vessels) and that of significant future risk (non-central macular oedema, surrogate markers of capillary non-perfusion or leakage). Readers are reminded of various classification systems described in literature, in particular, the need to be familiar with the retinopathy grading used in DR screening programmes and their correlation with clinical description of retinopathy. The ETDRS (Early Treatment for Diabetic Retinopathy Study) defined clinically significant macular oedema as macular oedema within 1 disc diameter (DD) of centre of macula, or lipid exudates within $1 \mathrm{DD}$ of centre of macula or retinal oedema $>1$ DD anywhere in macula. The OCT has become a routinely used technology to assess retinal thickness, and hence new classification of diabetic macular oedema (DMO) and treatment algorithms are OCT-based (Level A), taking into consideration whether the macular oedema is centre involving or not centre involving in addition to quantitative assessment of macular thickness and volume. It is recommended that the term maculopathy be confined to the screening programme while for clinical practice in hospital more specific descriptions of the features of macular oedema are used (Level A).

\section{Screening for DR}

NICE has recommended that all adult diabetic patients should have their eyes screened at the time of diagnosis and at least annually thereafter (Level A). Digital photography-based screening is established in the four nations of UK, with some variation in details on grading. It is aimed to improve identification of cases with sight threating retinopathy. Monitoring of screening programme performance against a set of quality assurance standards is the key to successful national screening programmes in all four nations (Level A). Children and adolescents with type I DM should undergo dilated fundus photography annually from age of 12, whereas those with type II DM should undergo dilated fundus photography annually from diagnosis (Level B). People with learning disability should not be excluded from the screening programme.

An ophthalmologist with expertise in DR should provide clinical leadership for the community-based screening programme and a medical retina expert should provide clinical leadership at the hospital eye service for clinical care provided in hospital (Level A). From public health point of view, DR-related visual loss accounted for $17.5 \%$ disability in working age population compared with $0.5 \%$ from AMD. Ophthalmologists can assist reducing this burden by developing strong links with local primary care and diabetology services to ensure that patients have effective integrated care plans. To address health inequalities, ophthalmologists can assist further public health research such as impact of socioeconomic status on DR and what steps can be taken to reduce inequalities in access and outcome (Level B).

\section{Management of diabetes}

In the management of DR, special attention is needed for the modifiable risk factors-such as the glycaemic control, hypertension, and lipids. Better glycaemic control reduces risks of microvascular complications. Improved $\mathrm{HbA1c}$ levels results in benefit of reducing progression of DR in both type I and type II diabetics and also offers long-lasting protective effect-'Metabolic memory'. It is recommended that personalised targets for $\mathrm{HbA1c}$ should be set (Level A). In patients with macular oedema, it is advisable to avoid Pioglitazone (Level B).

Similarly, better blood pressure control in diabetic patients reduces progression of DR; hence, a personalised blood pressure target should be set, which is adjusted for the severity of the retinopathy (Level B).

Antihypertensive medication with renin-angiotensin system blockade has preventive effect on DR in type I DM and protective therapeutic effect on progression of 
retinopathy in type II DM. Lowering of blood lipids is beneficial to diabetic maculopathy. Statins have modest protective effect but the Fenofibrates may have additional beneficial effect independent of lipid levels (Level B).

Retinopathy is common in pregnancy and there is small but significant risk of progression of retinopathy during pregnancy. Pregnant diabetic women should be offered digital retinal screening following their first antenatal check up and again at 28 weeks if the first assessment is normal. If any DR is present, additional retinal assessment should be performed at 16-20 weeks (Level A). Women with PDR in pregnancy should have ophthalmological follow-up for at least 6 months following the birth of the baby (Level B). DR should not be considered a contraindication to rapid optimisation of glycaemic control in women who present with a high $\mathrm{HbA1c}$ in early pregnancy (Level B).

\section{Management of DR}

Patients with just background DR can be managed in the community screening programme at appropriate intervals. Patients with preproliferative retinopathy characterised by signs of marked ischaemia-for example widespread blot haemorrhages, venous beading or presence of intraretinal microvascular abnormalities (IRMA) — need careful monitoring due to increased risk of progression to proliferative retinopathy. Closer follow-ups should be scheduled under the care of ophthalmologists, where interval between visits should be based on severity of retinal signs, systemic control, and patient factors (Level A). If there is concern about patient compliance and where retinopathy is progressive, retinal laser

photocoagulation may be considered (Level B).

PDR characterised by new vessels growth on optic disc or elsewhere on retina increases risk of vitreous haemorrhage $(\mathrm{VH})$, retinal fibrosis, and tractional retinal detachment with significant risk of vision loss. Pan retinal photocoagulation (PRP) laser treatment reduces risk of vision loss in PDR. Prompt, full PRP is recommended for patients with PDR (Level A). Laser treatment should be performed by ophthalmologists competent in technical skills as well as management of laser reactions (Level A). Multispot laser treatment strategies are effective in reducing risk of vision loss in PDR with better patient acceptance and potentially less risk of collateral damage and visual field loss. Intravitreal anti-VEGF injections can be useful in stabilising PDR, for example before vitrectomy or in presence of $\mathrm{VH}$ (Level B).

\section{Management of diabetic maculopathy}

Ophthalmic management of diabetic maculopathy depends on the location and extent of macular thickening (Figure 1). An OCT scan assists macular thickness assessment for management decisions. Patients with non centre-involving diabetic maculopathy can be treated with laser photocoagulation according to modified ETDRS criteria (Level A). Micropulse laser treatment to macula may achieve similar outcomes (Level B).

Patients with centre-involving macular oedema (central macular thickness $(\mathrm{CMT}) \geq 250 \mu \mathrm{m}$ and visual acuity in the region of $6 / 10-6 / 90$ ) would benefit most from anti-VEGF (Ranibizumab) treatment, which may be
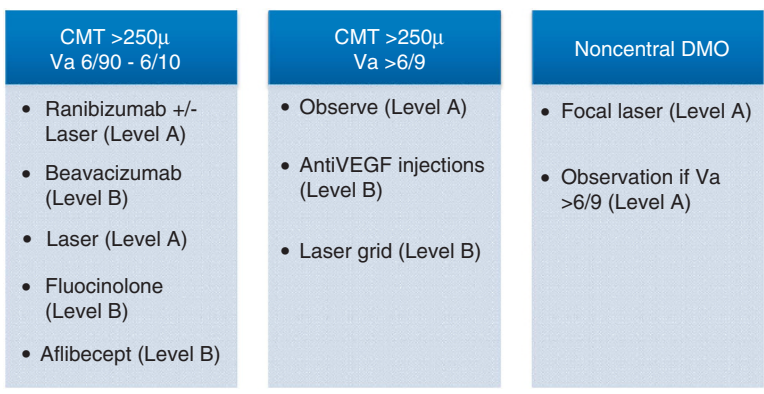

Figure 1 Treatment options for DMO.

combined with laser treatment (Level A). Where Ranibizumab is not available, Beavacizumab may be considered, in consultation with the patient (Level B). The Fluocinolone injection and Aflibercept injections offer other potential options (Level B), though these are currently not available for use in the NHS. In pseudophakic patients with DMO, intravitreal injection of Triamcinolone (Preservative-free where available) can be considered as an alternative option, combined with post-injection laser treatment to the macula (Level B).

Patients unwilling or unsuitable for injections can be offered macular laser treatment (Level A). If there is evidence of vitreomacular traction on the OCT scan, vitrectomy may be considered with or without adjunctive anti-VEGF/steroid treatment. Intravitreal Microplasmin injection can be useful in such cases (Level B). Patients with centre-involving macular oedema and good visual acuity $(>6 / 10)$ may be observed with close monitoring. Patients with poor visual acuity $(<6 / 90)$ may be observed especially if the macular oedema is long-standing and there is considerable macular ischaemia (Level B). Intravitreal Fluocinolone injection may be an option in long-standing DMO cases (Level B). In patients with marked reduction of vision but recent onset of DMO, discretionary treatment with anti-VEGF/ steroid injections or laser may be offered in consultation with patient if there is no significant macular ischaemia on fluorescein angiogram (Level C).

Patients needing laser treatment require three to four monthly follow-ups, whereas patients undergoing antiVEGF injections need monthly follow-up, at least in the first year (Level A). Patients with early maculopathy (but no CSMO) and background retinopathy (R1) can be followed up in ophthalmic imaging assessment clinics with colour images and OCT, at 4-6 monthly interval (Level B).

\section{Vitrectomy in diabetic eye disease}

In diabetic patients, vitreous haemorrhage $(\mathrm{VH})$ may commonly be due to progressive PDR or vitreo-retinal traction. Mild VH may be observed especially in the absence of active PDR. Additional retinal photocoagulation may be necessary if active PDR is found (Level A). Vitrectomy can be considered for patients with moderate to severe $\mathrm{VH}$, especially nonclearing $\mathrm{VH}, \mathrm{VH}$ in only eye, $\mathrm{VH}$ with retinal

detachment, and $\mathrm{VH}$ with ghost cell glaucoma (Level A). Traction retinal detachment especially involving macula can be relieved with vitrectomy combined with dissection of fibrovacular tissue and membrane peel. 
Proliferative retinopathy that is non- or poorly responding to full retinal laser treatment can be treated with pars plana vitrectomy. Young patients, especially with widespread fibrovascular proliferation benefit from vitrectomy (Level B). Similarly, DMO not responsive to treatment, especially cases with taut hyaloid face and those with vitreomacular traction can benefit from vitrectomy (Level B). Newer techniques of using antiVEGF injection concurrent with vitrectomy and use of microplasmin for chemical vitreolysis seem promising but need further evaluation (Level B). Early intervention with vitrectomy has been suggested to be of benefit in diabetic patients (Level B).

\section{Cataract}

Cataract is more common in diabetic patients but cataract surgery carries a higher risk of complications (OR 1.8) in this population (Level B). Cataract surgery should be performed with special attention to cortical clean up, avoiding lengthy procedures so as to reduce risks of uveitis and cystoid macular oedema (Level B). Serious postoperative complications are less frequent with modern phacoemulsification techniques. In patients with diabetes, endophthalmitis is likely to be more severe and leads to poorer visual outcome. Hence, surgeons need to pay specific attention to known surgical risk factors such as pre-existing ocular surface infection, wound construction, minimising tissue trauma, and avoiding surgical complications (Level A). Good diabetic control can help reduce postoperative complications (Level B).

Pre-existing macular oedema should be treated preoperatively, where possible. If this is not possible, intravitreal anti-VEGF or steroid injection (preferably preservative-free form) may be given at the conclusion of the cataract operation (Level A). Pre-existing proliferative retinopathy should be treated either preoperatively or at the conclusion of cataract procedure (Level A). DR may progress more rapidly following cataract surgery, hence it is advisable to monitor the eyes with pre-existing retinopathy closely in the postoperative period (Level A).

\section{Commissioning and set up for diabetic eye service} Diabetic eye clinics are getting busier with increasing diabetic population, maturing screening programmes, and new treatments, especially intravitreal injections. Ophthalmology departments are required to be engaged in provision of comprehensive care for the diabetic patients as well as collection of various data including data on outcomes (Level A). Dedicated lead ophthalmologist for diabetic service should be identified for hospital eye service in addition to lead ophthalmologist for diabetic screening programmes. Commissioning bodies, public health, and hospitals would need work collaboratively to ensure appropriate resources are available in eye departments to deliver optimum care for diabetic patients (Level A). The lead ophthalmologists will be involved in education and training of staff involved in clinical care of diabetic patients as well as providing advice and clinical leadership for screening programmes. Ophthalmologists with appropriate experience and training should be involved in treatment of diabetic patients especially for laser treatment (Level A). Diabetic eye clinics should have facility for ophthalmologists to deliver intravitreal injections in clean room. Dedicated diabetic eye clinic set up would have appropriate supporting staff consisting of nurses, administration-data clerk, photographer, and OCT technicians. Ophthalmologists supported by such arrangements can facilitate development of pathways where patients without severe retinopathy and treated stable retinopathy can be seen in virtual clinics combining fundus photography and spectral domain OCT scans (Level B).

Management of DR and maculopathy has changed significantly with the development of new technology and treatment options. Research in this field is growing, and as a result the clinical practice for DR will continue to evolve.

\section{Guidelines}

The guidelines described recommendation levels as follows: Level A: where strength of evidence was universally agreed; Level B: where the probability of benefit to the patient outweighed the risks; Level C: where it was recognised that there was difference of opinion as to the likely benefit to the patient and decision to treat would be based after discussion with the patient. Full guidelines are available at www.rcophth.ac.uk/clinicalguidelines

\section{Contributors}

Ghanchi F, Bailey C, Chakravarthy U, Cohen S, Dodson P, Gibson J, Menon G, Muqit M, Pilling R, Olson J, Prasad S, Scanlon P, Stanga P, Vafidis G, Wright A and Wykes W.

F Ghanchi ${ }^{1}$ and the Diabetic Retinopathy Guidelines Working Group ${ }^{2}$

${ }^{1}$ Bradford Teaching Hospitals, Bradford, UK

${ }^{2}$ The members of the Diabetic Retinopathy Guidelines Working Group who participated are listed at the end of the article.

F Ghanchi, Bradford Teaching Hospitals, Duckworth Lane, Bradford BD9 6RJ, UK. E-mail: Faruque.ghanchi@bthft.nhs.uk

Eye (2013) 27, 285-287; doi:10.1038/eye.2012.287;

published online 11 January 2013 